\title{
Detailed analysis of a superficial CD34-positive fibroblastic tumor: A case report and review of the literature
}

\author{
KENSAKU YAMAGA $^{1}$, AKIHIRO FUJTAA ${ }^{1}$, MARI OSAKI ${ }^{1}$, SATOSHI KUWAMOTO ${ }^{2}$, \\ NAOKO ISHIGURO ${ }^{3}$, TADAHITO YAMAMOTO $^{4}$ and HIDEKI NAGASHIMA ${ }^{1}$
}

\author{
${ }^{1}$ Department of Orthopedic Surgery, Faculty of Medicine, Tottori University, Yonago, Tottori 683-8504; \\ ${ }^{2}$ Department of Pathology, Tottori University Hospital, Yonago, Tottori 683-8504; ${ }^{3}$ Department of Pathobiological Science and \\ Technology, Faculty of Medicine, Tottori University, Yonago, Tottori 683-8503; \\ ${ }^{4}$ Department of Orthopedic Surgery, Matsue City Hospital, Matsue, Shimane 690-0045, Japan
}

Received January 11, 2017; Accepted March 30, 2017

DOI: $10.3892 / 01.2017 .6636$

\begin{abstract}
Superficial cluster of differentiation (CD)34-positive fibroblastic tumor (SCPFT) is a rare mesenchymal neoplasm of borderline malignancy. It is characterized by a superficial location, marked cellular pleomorphism, an extremely low incidence of mitotic figures, and strong CD34 immunohistochemical positivity. As SCPFT is a recently described neoplasm, its characteristics are yet to be fully elucidated. To the best of our knowledge, no detailed studies regarding the imaging findings and cytogenetic analyses of SCPFTs exist. The present study describes a typical case of an 18-year-old man who developed an SCPFT measuring $87 \times 70 \times 80 \mathrm{~mm}$ in the subcutaneous adipose tissue of his right thigh. Computed tomography (CT) revealed a well-marginated tumor without calcification, and the enhancement on CT was weak. The tumor demonstrated abnormal uptake on 2-(18F) fluoro-2-deoxy-D-glucose positron emission tomography (PET), with a maximum standardized uptake value of 2.57 . Magnetic resonance imaging (MRI) revealed a clearly defined tumor that exhibited homogeneous low signal intensity on T1-weighted imaging and high signal intensity on T2-weighted imaging, with small lobulated structures. Histopathologically, the tumor was composed of irregular spindle-to-oval-shaped cells with eosinophilic glassy cytoplasm and hyperchromatic, bizarre and pleomorphic nuclei that frequently exhibited intranuclear pseudoinclusions. Immunohistochemically, the tumor cells were diffusely and strongly positive for CD34. The Mindbomb E3 ubiquitin protein ligase 1 labeling index was $8.6 \%$. Ultrastructurally, the tumor cells exhibited irregular
\end{abstract}

Correspondence to: Dr Kensaku Yamaga, Department of Orthopedic Surgery, Faculty of Medicine, Tottori University, 36-1 Nishi-cho, Yonago, Tottori 683-8504, Japan

E-mail:kyamaga@med.tottori-u.ac.jp

Key words: cluster of differentiation 34, fibroblastic tumor, superficial, positron emission tomography, chromosome, translocation or convoluted nuclei with abundant euchromatin-prominent nucleoli. The cytoplasmic organelles consisted of scattered, abundant rough endoplasmic reticulum, mitochondria, lysosomes, ribosomal rosettes and aggregated lipid globules. Of 18 metaphase cells identified, 2 demonstrated translocation between chromosomes 2 and 5 in cytogenetic studies. To the best of our knowledge, this is the first study describing imaging data (CT, MRI and PET-CT) and chromosomal aberrations for SCPFT.

\section{Introduction}

Superficial cluster of differentiation (CD)34-positive fibroblastic tumors (SCPFTs), first identified by Carter et al (1) are rare mesenchymal neoplasms of intermediate (borderline) malignancy. As they were only described recently, their characteristics are yet to be entirely elucidated. They were cured by surgery and had a good outcome (1). Previous studies of SCPFTs have lacked imaging data or cytogenetic analyses of this tumor type (1-4).

The present study describes a patient with SCPFT located in the subcutaneous adipose tissue of the thigh of an 18-year-old man. The imaging, histopathological and ultrastructural data, and the cytogenetic study results are discussed. Aside from histopathology and immunohistochemistry, the examination of this patient encompassed computed tomography (CT), magnetic resonance imaging (MRI), and positron emission tomography (PET)-CT, and cytogenetic analysis to identify chromosomal aberrations. Informed consent for publication of these data was obtained from the patient.

\section{Case report}

Clinical and imaging data. An 18-year-old Japanese male noticed a painless mass measuring $\sim 3 \mathrm{~cm}$ in the medial aspect of his right distal thigh, without history of trauma. He visited Matsue City Hospital (Matsue, Japan) in April, 2015, where the mass was initially diagnosed as an epidermoid cyst. Surgical resection of the mass was planned, but was cancelled subsequent to the patient developing a cold. The patient was subsequently lost to follow-up for 18 months, following which 
he revisited Matsue City Hospital as the mass had continued to grow. At that point, a soft tissue sarcoma was suspected. He was referred to Tottori University Hospital (Yonago, Japan) in October, 2016 for evaluation and treatment. Physical examination revealed a $9-\mathrm{cm}$, hard, elastic mass in his distal thigh without tenderness or warm sensation, but with varicosis (Fig. 1). Medical and family history were unremarkable. The white blood cell count $\left(3.8-8.8 \times 10^{3} / \mu 1\right)$ and the levels of C-reactive protein $(<0.15 \mathrm{mg} / \mathrm{dl})$, alkaline phosphatase (106-322 U/1) and lactate dehydrogenase (124-222 U/l) were normal. CT revealed a well-marginated tumor without calcification in the subcutaneous adipose tissue, and enhanced CT revealed weak enhancement within the lesion (Fig. 2A and B). The tumor demonstrated abnormal uptake on $2-\left({ }^{18} \mathrm{~F}\right)$ fluoro-2-deoxy-D-glucose (18F-FDG) PET, with a maximum standardized uptake value of 2.57 (Fig. 2C). The MRI scan revealed a clearly defined tumor measuring $87 \times 70 \times 80 \mathrm{~mm}$ and located in the subcutaneous adipose tissue; however, part of the tumor margin appeared to slightly infiltrate the subcutaneous adipose tissue (Fig. 3). Although the tumor appeared to be in close proximity to the vastus medialis and sartorius muscles, it did not infiltrate them. The tumor exhibited homogenous low signal intensity on T1-weighted imaging (Fig. 3A) and high signal intensity on T2-weighted imaging, with small lobulated structures (Fig. 3B). Mild enhancement was observed throughout the tumor, while strong enhancement was evident at the periphery of the small lobulated structures (Fig. 3C). No distant or lymph node metastasis was observed.

Based on the aforementioned clinical data and imaging examinations, the mass was diagnosed as a malignant tumor of the soft tissue; such tumors include synovial sarcoma, alveolar soft part sarcoma, and epithelioid sarcoma. Core needle biopsy was performed for histopathological diagnosis. Although the tumor was suspected to be an SCPFT, a definite diagnosis was not initially available. The differential diagnosis was pleomorphic sarcoma. Thereafter, wide resection was performed.

Pathological data (surgical specimen). Grossly, the tumor was well-circumscribed and confined to the superficial fibroadipose tissues. It exhibited a yellow and fleshy appearance with small lobulated structures (Fig. 4).

For light microscopic examination (magnification x100 to $\mathrm{x} 400$ ), the tumor was fixed in $10 \%$ buffered-formalin for $24 \mathrm{~h}$ at room temperature, and embedded in paraffin wax. Serial sections, $4 \mu \mathrm{m}$ thick, were stained using hematoxylin (Mayer's hematoxylin solution) and $0.5 \%$ eosin. Histopathologically, the tumor was well-circumscribed and had not infiltrated into adjacent tissues. It was composed of irregular spindle-to-oval-shaped cells, with eosinophilic glassy cytoplasm and hyperchromatic, bizarre and pleomorphic nuclei that frequently demonstrated intranuclear pseudoinclusions. Tumor cells were arranged in fascicles and sheets, with a focal myxoid change in the background. A small number of inflammatory cells, predominantly lymphocytes, were identified throughout the lesion. Mitotic figures were rarely observed (2/50 high-powered fields), and atypical mitoses were not observed (Fig. 5A-F). No necrosis was observed.

For immunohistochemical analysis, paraffin-embedded tumor tissue specimens were cut into $4-\mu \mathrm{m}$ sections, then dewaxed in xylene, rehydrated through a graded series of
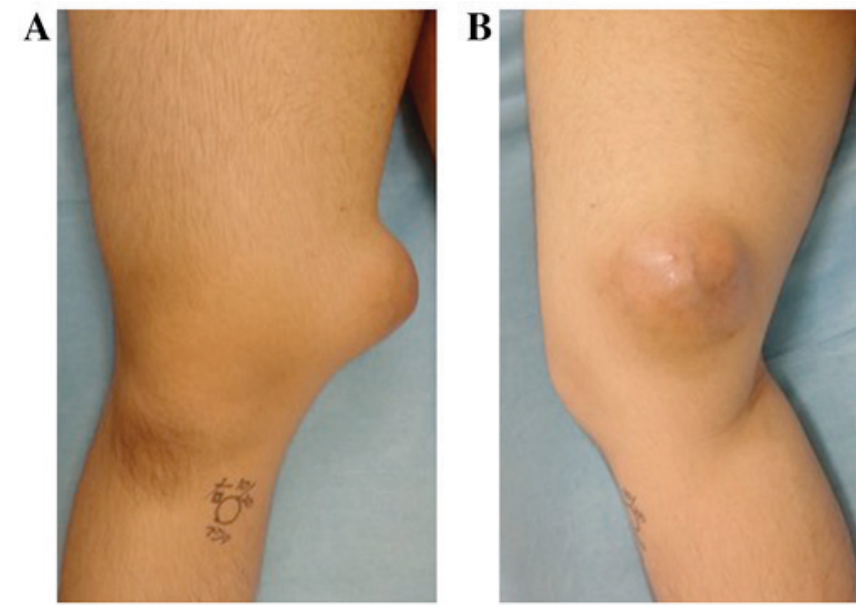

Figure 1. Physical examination revealed a $9-\mathrm{cm}$, hard, elastic mass in the distal thigh of the patient without tenderness or warm sensation, but with varicosis. (A) Frontal view. (B) Medial view.

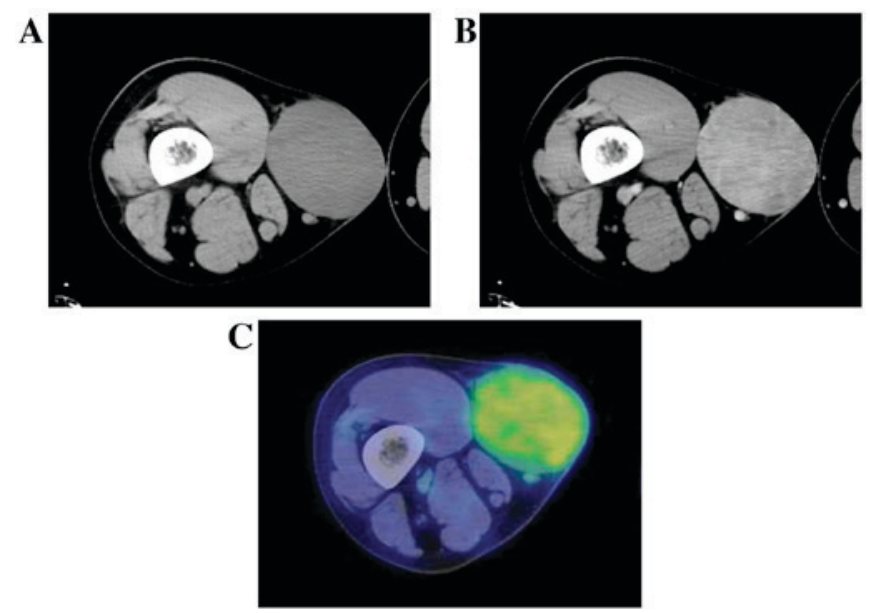

Figure 2. (A) Plain CT revealed that the tumor was located in the subcutaneous adipose tissue, and was well-circumscribed.(B) Enhanced CT revealed diffuse and weak enhancement in the tumor. (C) The maximum standardized update value of the tumor was 2.57 on positron emission tomography-CT. CT, computed tomography.

ethanol solutions and rinsed in distilled water for $5 \mathrm{~min}$. Endogenous peroxidase activity was blocked by $2 \%$ hydrogen peroxide in methanol at room temperature for $30 \mathrm{~min}$. Subsequent to rinsing with PBS, the sections were incubated with blocking serum ( $2 \%$ fetal bovine serum) at room temperature for $20 \mathrm{~min}$ and incubated at $4^{\circ} \mathrm{C}$ overnight with each primary antibody. Immunohistochemically (by light microscope with magnification, $x 100$ to $\times 200$ ), the tumor cells were diffuse and strongly positive for CD34 (catalog no. 413111, prediluted, ready to use; Nichirei, Tokyo, Japan; Fig. 5G) and negative for CD31 (catalog no. M0823, prediluted, ready to use; Dako; Agilent Technologies, Inc., Santa Clara, CA, USA), desmin (catalog no. 413651, prediluted, ready to use; Nichirei Bioscience, Tokyo, Japan), $\alpha$-smooth muscle actin (catalog no. M0851, dilution, 1:100; Dako; Agilent Technologies, Inc.), S-100 protein (catalog no. 422091, prediluted, ready to use; Nichirei Bioscience), cytokeratin (AE1/AE3; catalog no. 412811, prediluted, ready to use; Nichirei Bioscience), epithelial membrane antigen (catalog no. M0613, prediluted, ready to use; Dako; 

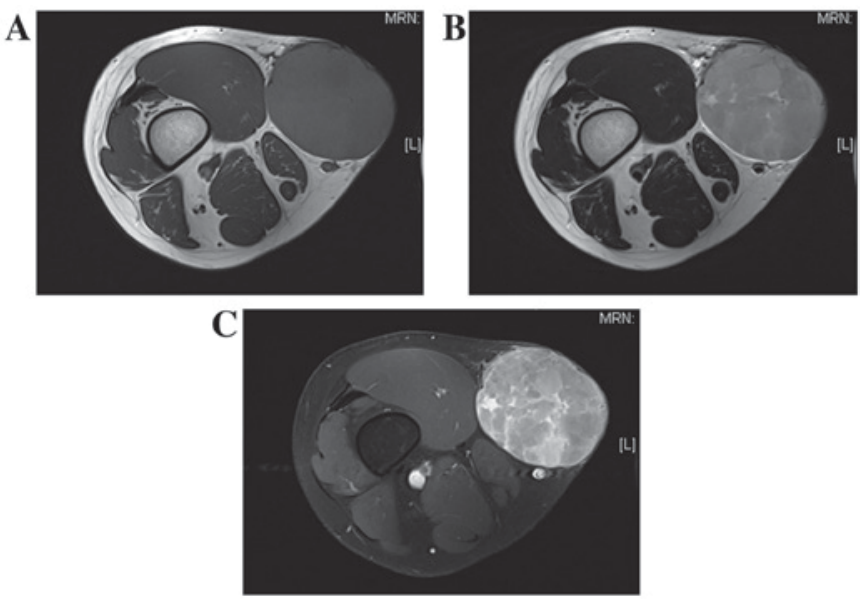

Figure 3. Representative horizontal magnetic resonance images. The tumor was located in the subcutaneous adipose tissue, close to the vastus medialis and sartorius muscles, and exhibited a relatively well-defined margin. (A) T1-weighted imaging demonstrated low signal intensity in the mass. (B) T2-weighted imaging revealed high signal intensity, with small lobulated structures. (C) Mild enhancement was observed throughout the tumor, and strong enhancement was observed at the periphery of the small lobulated structures.

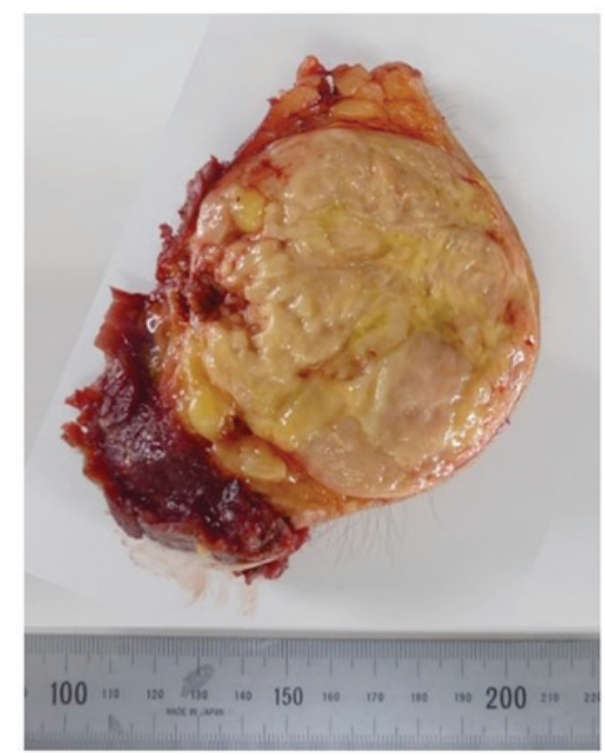

Figure 4. Cut section of tumor. The tumor was well-circumscribed and had a yellow, fleshy appearance with small lobulated structures.

Agilent Technologies, Inc.), signal transducer and activator of transcription 6 (STAT6; catalog no. sc-29497, dilution, 1:300; Santa Cruz Biotechnology, Inc., Dallas, TX, USA), B-cell lymphoma-2 (catalog no. 413141, prediluted, ready to use; Nichirei Bioscience) and c-Kit (catalog no. A4502, dilution 1:100; Dako; Agilent Technologies, Inc.). Integrase interactor 1 protein (INI1; catalog no. A301-087A dilution, 1:250; Bethyl Laboratories, Montgomery, TX, USA) was expressed throughout the lesion. The Mindbomb E3 ubiquitin protein ligase 1 (MIB1; catalog no. M7240, dilution, 1:100; Dako; Agilent Technologies, Inc.) labeling index in the tumor was $8.6 \%$ (Fig. $5 \mathrm{H}$ ).

Ultrastructural examination. For transmission electron microscopic study, the tumor was fixed overnight at $4^{\circ} \mathrm{C}$

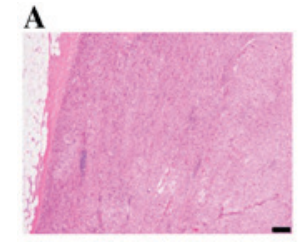

D
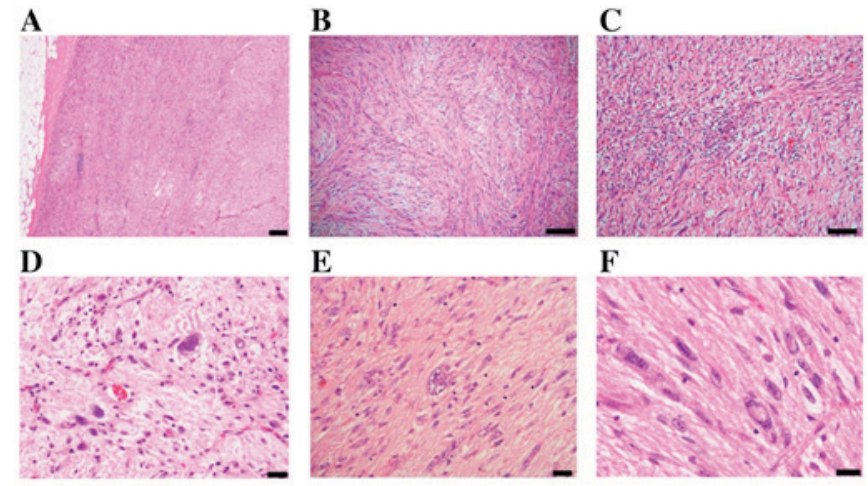

G

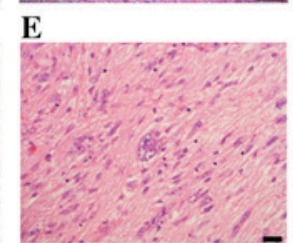

F

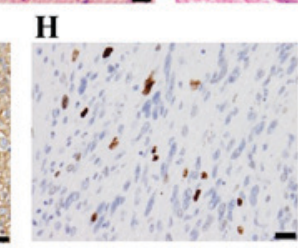

Figure 5. Histological and immunohistochemical staining of the tumor. Staining methods included (A-F) hematoxylin and eosin, and antibodies against (G) CD34 and (H) MIB-1. (A) The lesion was well-circumscribed and had not invaded the subcutaneous tissue. (B-F) The tumor was composed of fascicles or sheets of pleomorphic spindle-to-oval-shaped cells that exhibited irregularly hyperchromatic, bizarre and pleomorphic nuclei with frequent intranuclear pseudoinclusions and rare mitotic figures. (C) A small number of inflammatory cells were identified throughout the lesion. (D) Partial myxoid stroma was observed. (G) Immunohistochemically, CD34 was strongly and diffusely expressed in almost all of the tumor cells. (H) The MIB-1 labeling index was $8.6 \%$. Scale bars: (A) $200 \mu \mathrm{m}$; (B and C) $100 \mu \mathrm{m}$; (D-H) $20 \mu \mathrm{m}$.CD, cluster of differentiation; MIB-1, Mindbomb E3 ubiquitin protein ligase 1.

in $2.5 \%$ glutaraldehyde in $0.1 \mathrm{M}$ cacodylate buffer, $\mathrm{pH} 7.4$, followed by post-fixation for $1-2 \mathrm{~h}$ in $0.1 \mathrm{M}$ cacodylate-buffered $1 \%$ osmium tetraoxide. Subsequent to dehydration in a series of graded ethanol and n-butyl glycidyl ether solutions, cells were embedded in epoxy resin and allowed to set for $48 \mathrm{~h}$ at $60^{\circ} \mathrm{C}$. Ultrathin sections were stained with uranyl acetate and lead citrate, and examined with an electron microscope. The tumor consisted of spindle-to-oval shaped cells without intercellular junctions. The cells had irregular or convoluted nuclei with abundant, euchromatin-prominent nucleoli. The cytoplasmic organelles consisted of scattered, abundant and rough endoplasmic reticulum, mitochondria, lysosomes, ribosomal rosettes and aggregated lipid globules (Fig. 6).

Cytogenetic studies. Cytogenetic analysis was performed on the surgical specimen. The tissues were cut into pieces and transferred to culture solution. Cell culture and G-band karyotyping were performed by SRL Inc. (Tokyo, Japan). Of the 18 metaphase cells available for analysis, 14 were of normal (46,XY) karyotype, 2 cells had a karyotype of $\mathrm{t}(2 ; 5)(\mathrm{q} 31 ; \mathrm{q} 31)$, 1 had a karyotype of 42,Y,-X,-10,-18,-19, $\operatorname{add}(19)(\mathrm{p} 13)$, and 1 had a karyotype of $\mathrm{t}(7 ; 14)(\mathrm{q} 21 ; \mathrm{q} 24)$ (Fig. 7).

The tumor was finally diagnosed definitively as an SCPFT of the thigh based on clinical, histological and immunohistochemical data. In particular, the results that CD34 was strongly positive on immunohistochemistry, and that the tumor site was superficial, assisted with the diagnosis. The postoperative course ( 5 months subsequent to surgery) has been uneventful, with no evidence of recurrence or metastasis. 
Table I. Clinical outcomes reported in previous studies of superficial CD34-positive fibroblastic tumors.

\begin{tabular}{lccclll}
\hline Author, year & No. & $\begin{array}{c}\text { No. with } \\
\text { available } \\
\text { follow-up } \\
\text { data }\end{array}$ & $\begin{array}{c}\text { Median } \\
\text { follow-up } \\
\text { duration } \\
\text { (months) }\end{array}$ & $\begin{array}{c}\text { No. with local } \\
\text { recurrences } \\
\text { and distant } \\
\text { metastases }\end{array}$ & Outcome & (Refs.) \\
\hline Carter et al, 2014 & 18 & 13 & 24 & $\begin{array}{l}\text { 1 (regional } \\
\text { lymph node } \\
\text { metastasis) }\end{array}$ & 1 ANED & 12 CDF \\
Hendry et al, 2015 & 2 & 2 & 3 & None & All CDF & $(4)$ \\
Wada et al, 2016 & 1 & 1 & 3 & None & CDF & $(2)$ \\
Lao et al, 2017 & 11 & 11 & 6 & None & All CDF & $(3)$ \\
Li et al, 2016 & 1 & 1 & 24 & None & CDF & (5) \\
Present study & 1 & 1 & 5 & None & CDF & - \\
\hline
\end{tabular}

No., number of cases; ANED, alive no evidence of disease; CDF, continuous disease free.

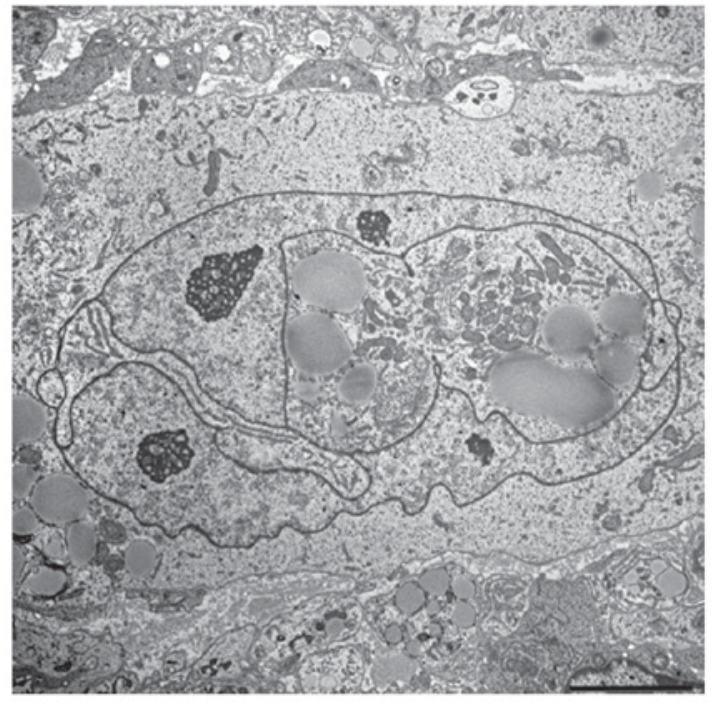

Figure 6. Transmission electron microscopy. Ultrastructural examination revealed that the tumor cells possessed irregular or convoluted nuclei with abundant euchromatin-prominent nucleoli. In the cytoplasm, aggregated lipid globules were observed. Scale bar, $5 \mu \mathrm{m}$.

\section{Discussion}

When this tumor type was first described in 2014 in a series of 18 cases, Carter et al (1) proposed the descriptive term SCPFT based on clinical, morphological and immunochemical data. To date, only five case series of SCPFT have been described (1-5), in which almost all tumors were described histopathologically and immunohistochemically. Only one study, that of Li et al (5), described imaging data, namely T1-weighted MRI findings. To the best of our knowledge, CT and PET-CT features of SCPFT have not been described prior to the present study.

The available studies on SCPFT described certain shared characteristics: A fibroblastic tumor of the superficial soft tissues with cellular pleomorphism, an extremely low mitotic rate, and strong diffuse CD34 positivity (1-5). In their 18-case

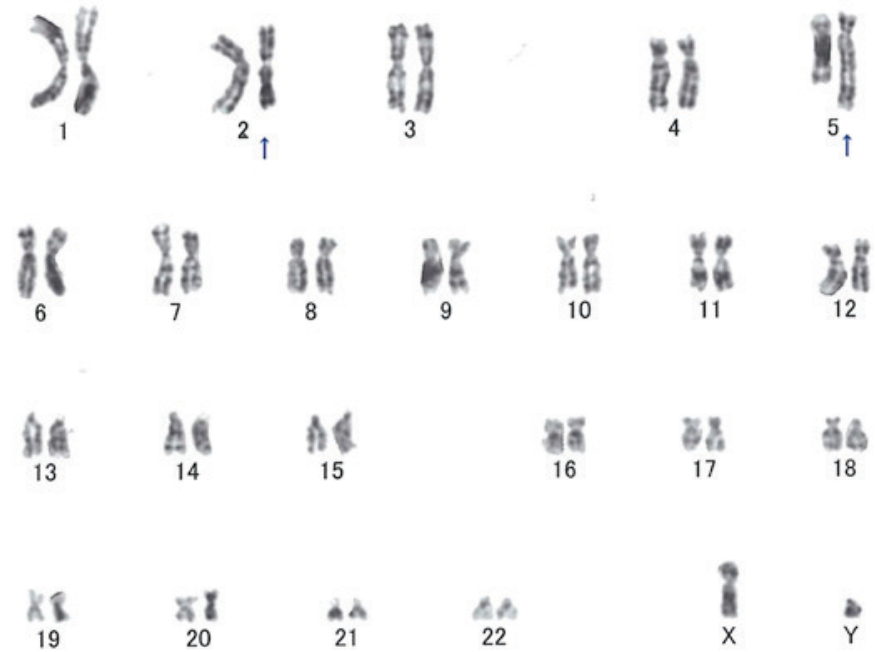

Figure 7. Tumor cell chromosomes exhibit a translocation between chromosomes 2 and 5 (arrows)

series, Carter et al (1) described SCPFTs as slow-growing masses that are present for at least 1 year prior to diagnosis, that range in size from 1.5 to $10 \mathrm{~cm}$ (mean, $4.1 \mathrm{~cm}$ ), and that most commonly afflict adults (age range, 20-76 years; median, 38 years) of any sex (10 males and 8 females). In two-thirds of the cases, the tumors occurred in the lower limbs, including the thighs. All of the tumors showed confinement to the superficial fibroadipose tissues, and absent or minimal involvement of the subjacent muscle was observed. There was no history of a previous cutaneous neoplasm at the same location in any of the patients. Clinical follow-up data (median duration, 24 months; range, 1-104 months) were available for 13 of the 18 patients (72\%). Of these 13 patients, 12 were alive with no evidence of disease when the study was published, and 1 patient with a tumor in the thigh experienced metastasis to an external iliac lymph node 7 years after marginal excision of the primary tumor, but was alive at the end of the follow-up. Table I describes the clinical outcomes reported in previous studies. 
On microscopic examination, the tumors in all patients described by Carter et al (1) were confined to the superficial fibroadipose tissues and were composed of spindle-to-epithelioid cells with an abundant granular or glassy cytoplasm arranged in fascicles and sheets. Marked pleomorphisms and a low incidence of mitotic figures were observed in all patients. On immunohistochemical examination, all tumors exhibited strong, diffuse CD34 positivity. Limited cytokeratin expression was observed in the neoplastic cells of 11 out of 16 assessed cases. Lao et al (3) suggested that it is not uncommon to misdiagnose SCPFT as a pleomorphic dermal neoplasm (particularly atypical fibroxanthoma, pleomorphic dermal sarcoma, or undifferentiated pleomorphic sarcoma/malignant fibrous histiocytoma) due to this marked pleomorphism.

The present study is a typical case of SCPFT based on the histopathological data, which are consistent with previous studies. The specific findings that CD34 was strongly positive on immunohistochemistry, and that the tumor site was superficial, assisted with the diagnosis. Although PET imaging findings for this type of tumor have not been discussed previously, the maximum standard update value on 18F-FDG PET was 2.57 in the patient of the present study; similar values are frequently observed in soft tissue tumors of intermediate malignancy (6-8). The PET findings in the current patient are considered consistent with an intermediate malignancy as described by Carter et al (1). On ultrastructural examination, the tumor cells in the present case exhibited a tendency towards fibroblast differentiation, consistent with the findings described by Hendry et al (4). It is unclear whether this feature is specific to all SCPFTs, as Hendry et al (4) were the only group aside from ours to perform ultrastructural examination. Cytogenetic studies in the present case revealed a chromosomal translocation of $\mathrm{t}(2 ; 5)(\mathrm{q} 31 ; \mathrm{q} 31)$, according to the International System for Human Cytogenetic Nomenclature criteria (9). Juvenile-onset soft tissue sarcomas are often characterized by disease-specific fusion genes resulting from translocations, such as $\mathrm{t}(\mathrm{X} ; 18)$ in synovial sarcomas, $\mathrm{t}(12 ; 22)$ or $\mathrm{t}(2 ; 22)$ in clear cell sarcomas, $\mathrm{t}(2 ; 13)$ in alveolar rhabdomyosarcoma, and $\mathrm{t}(\mathrm{X} ; 17)$ in alveolar soft part sarcomas (10-13). Additionally, Carter et al (1) suggested that SCPFT most commonly affects young to middle-aged adults. Therefore, we hypothesized that the $\mathrm{t}(2 ; 5)(\mathrm{q} 31 ; \mathrm{q} 31)$ translocations observed in the present case suggest the existence of a chromosomal aberration specific to SCPFT. In previous studies, 3 patients with translocation-associated soft tissue tumors involving $2 \mathrm{q} 31$, including 2 patients with synovial sarcoma and 1 with lipoblastoma, and 1 patient with translocation-associated embryonal rhabdomyosarcoma involving 5q31, were documented (14-17).

Clinically, surgeons who are not orthopedic oncologists may perform unplanned excisions for such tumors owing to their small sizes and superficial locations; they may not accurately diagnose SCPFT owing to its rarity. As mentioned, the tumor of the present patient was initially diagnosed as an epidermoid cyst. Surgical resection was planned, and the patient almost underwent surgery at a nearby hospital 18 months prior to visiting Tottori University Hospital.

In summary, the present study described a typical case of SCPFT in the thigh of an 18-year-old man. To the best of our knowledge, this is the first study of an SCPFT in which imaging data (CT, MRI, and PET-CT) and chromosomal aberrations are described in detail for this type of tumor. It was identified that $\mathrm{t}(2 ; 5)(\mathrm{q} 31 ; \mathrm{q} 31)$ may be a disease-specific chromosomal aberration in SCPFT. As additional characteristics of SCPFTs remain to be identified, it is important to accumulate more patients and review long-term follow-up data. This will assist in improving recognition of these tumors, and avoid misdiagnosing them as high-grade sarcomas.

\section{Acknowledgements}

The authors would like to thank Mr. T. Horie of the Technical Department, Division of Medical Science, Tottori University (Tottori, Japan), for his excellent technical assistance, and Dr S. Oda of the Department of Pathology and Experimental Medicine, Okayama University (Okayama, Japan) for performing STAT6 immunostaining.

\section{References}

1. Carter JM, Weiss SW, Linos K, DiCaudo DJ and Folpe AL: Superficial CD34-positive fibroblastic tumor: Report of 18 cases of a distinctive low-grade mesenchymal neoplasm of intermediate (borderline) malignancy. Mod Pathol 27: 294-302, 2014.

2. Wada N, Ito T, Uchi H, Nakahara T, Tsuji G, Yamada Y, Oda Y and Furue M: Superficial CD34-positive fibroblastic tumor: A new case from Japan. J Dermatol 43: 934-936, 2016.

3. Lao IW, Yu L and Wang J: Superficial CD34-positive fibroblastic tumor: A clinicopathological and immunohistochemical study of an additional series. Histopathology 70: 394-401, 2017.

4. Hendry SA, Wong DD, Papadimitriou J, Robbins P and Wood BA: Superficial CD34-positive fibroblastic tumour: Report of two new cases. Pathology 47: 479-482, 2015.

5. Li W, Molnar SL, Mott M, White E and De Las Casas LE: Superficial CD34-positive fibroblastic tumor: Cytologic features, tissue correlation, ancillary studies, and differential diagnosis of a recently described soft tissue neoplasm. Diagn Cytopathol 44: 926-930, 2016.

6. Ioannidis JP and Lau J: 18F-FDG PET for the diagnosis and grading of soft-tissue sarcoma: A meta-analysis. J Nucl Med 44: 717-724, 2003.

7. Tian R, Su M, Tian Y, Li F, Li L, Kuang A and Zeng J: Dual-time point PET/CT with F-18 FDG for the differentiation of malignant and benign bone lesions. Skeletal Radiol 38: 451-458, 2009.

8. Aoki J, Watanabe H, Shinozaki T, Takagishi K, Tokunaga M, Koyama Y, Sato N and Endo K: FDG-PET for preoperative differential diagnosis between benign and malignant soft tissue masses. Skeletal Radiol 32: 133-138, 2003.

9. Mitelman F (ed); International Standing Committee on Human Cytogenetic Nomenclature: ISCN 1995: an international system for human cytogenetic nomenclature (1995): recommendations of the International Standing Committee on Human Cytogenetic Nomenclature, Memphis, Tennessee, USA, October 9-13, 1994. Karger, Basel, 1995.

10. Suurmeijer AJH, de Bruijn D, Geurts van Kessel A and Miettinen MM: Synovial sarcoma. In: World Health Organization Classification of Tumours. Soft Tissue and Bone. 4th edition. Fletcher CDM, Bridge JA, Hogendoorn PCW and Mertens F (eds). IARC Press, Lyon, pp213-215, 2013.

11. Antonescu CR: Clear cell sarcoma of soft tissue. In: World Health Organization Classification of Tumours. Soft Tissue and Bone. 4th edition. Fletcher CDM, Bridge JA, Hogendoorn PCW and Mertens F (eds). IARC Press, Lyon, pp221-222, 2013.

12. Parham DM and Barr FG: Alveolar rhabdomyosarcoma. In: World Health Organization Classification of Tumours. Soft Tissue and Bone. 4th edition. Fletcher CDM, Bridge JA, Hogendoorn PCW and Mertens F (eds). IARC Press, Lyon, pp130-132, 2013.

13. Ordonez NG and Ladanyi M: Alveolar soft part sarcoma. In: World Health Organization Classification of Tumours. Soft Tissue and Bone. 4th edition. Fletcher CDM, Bridge JA, Hogendoorn PCW and Mertens F (eds). IARC Press, Lyon, pp218-220, 2013. 
14. Panagopoulos I, Mertens F, Isaksson M, Limon J, Gustafson P, Skytting B, Akerman M, Sciot R, Dal Cin P, Samson I, et al: Clinical impact of molecular and cytogenetic findings in synovial sarcoma. Genes Chromosomes Cancer 31: 362-372, 2001.

15. Przybyl J, Sciot R, Rutkowski P, Siedlecki JA, Vanspauwen V, Samson I and Debiec-Rychter M: Recurrent and novel SS18-SSX fusion transcripts in synovial sarcoma: Description of three new cases. Tumour Biol 33: 2245-2253, 2012.
16. Yoshida H, Miyachi M, Ouchi K, Kuwahara Y, Tsuchiya K, Iehara T, Konishi E, Yanagisawa A and Hosoi H: Identification of COL3A1 and RAB2A as novel translocation partner genes of PLAG1 in lipoblastoma. Genes Chromosomes Cancer 53: 606-611, 2014.

17. Roberts I, Foster N, Nacheva E and Coleman N: Paint-assisted microdissection-FISH: Rapid and simple mapping of translocation breakpoints in the embryonal rhabdomyosarcoma cell line RD. Cytometry A 58: 177-184, 2004. 